\title{
Solution Structures of Purine Base Analogues 9-deazaguanine and 9-deazahypoxanthine
}

\author{
Vishakha Karnawat and Mrinalini Puranik ${ }^{*}$ \\ ${ }^{1}$ Indian Institute of Science Education and Research, Pune-411008, India
}

Supplementary Information

Manuscript submitted to: Journal of Biomolecular Structure and Dynamics

*Corresponding Author:

Dr. Mrinalini Puranik, Mendeleev Building, Indian Institute of Science Education and Research,

Pune-411008, Maharashtra, India

Phone: +91-735 069 4600; Email: mrinalini@iiserpune.ac.in 


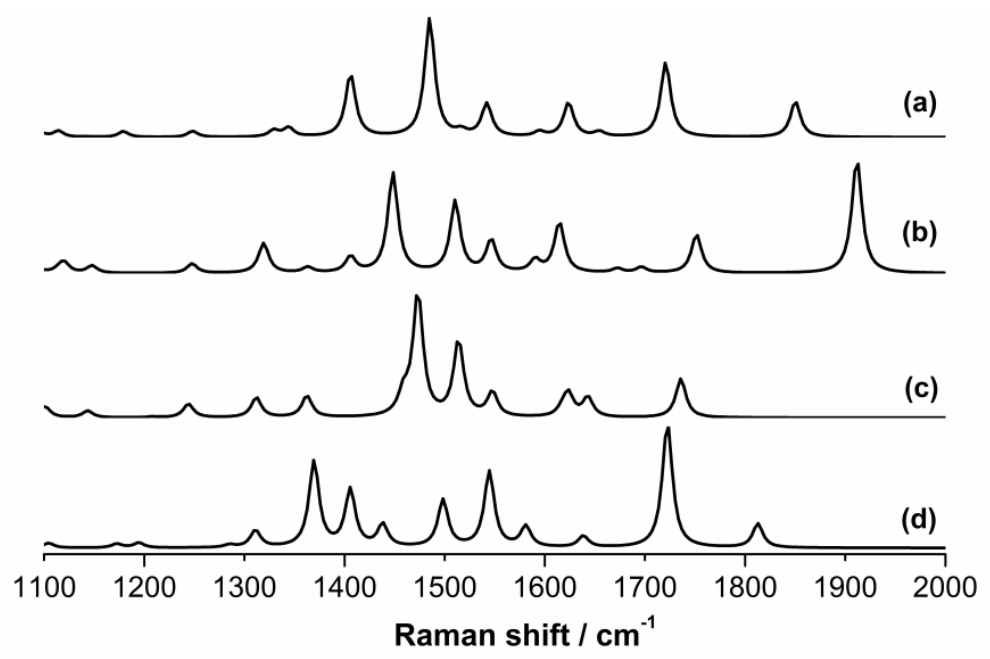

Figure S1: DFT (B3LYP/6-31G (d, p) computed spectra of (a) neutral 9DAG, (b) N3 protonated, (c) N1 deprotonated, (d) N7 deprotonated. N7 deprotonated shows weak carbonyl stretch at 1813 $\mathrm{cm}^{-1}$ whereas in $\mathrm{N} 1$ deprotonated, the carbonyl stretch is completely disappeared. 


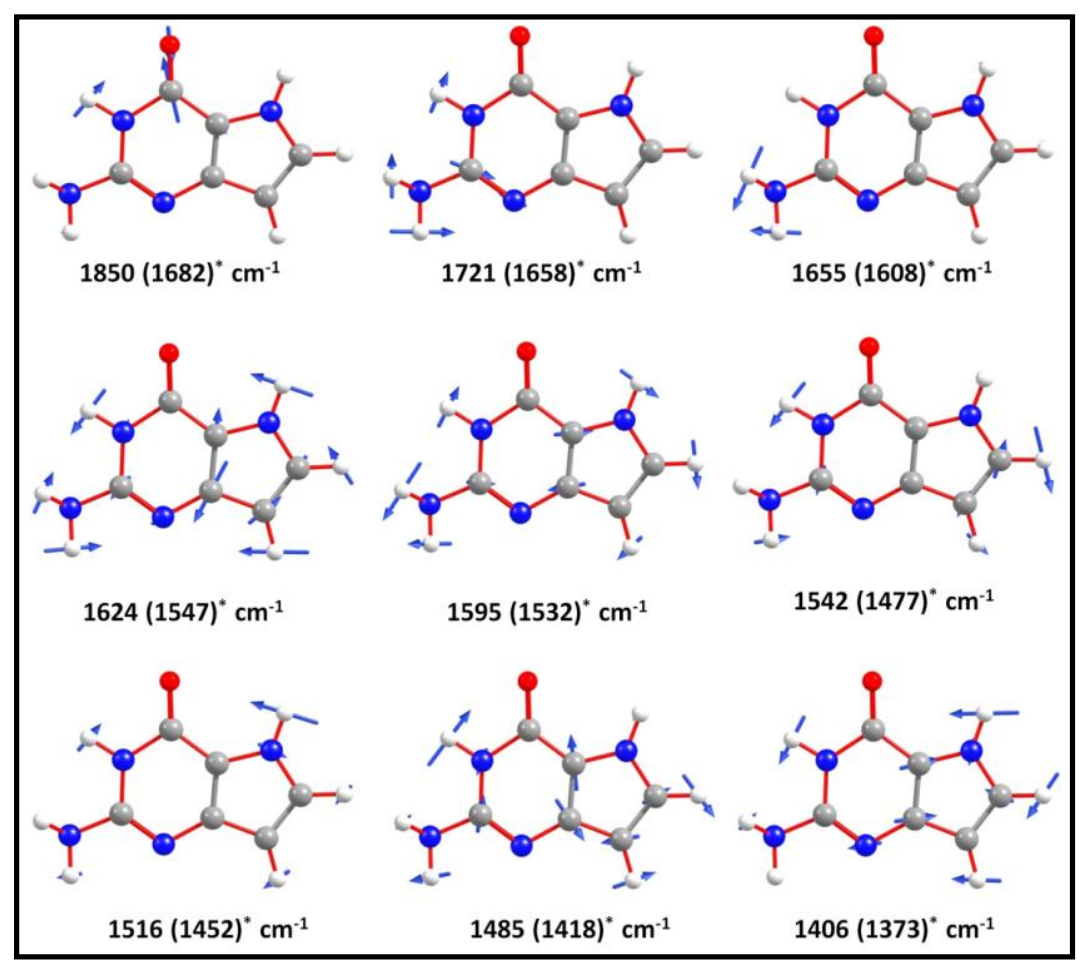

Figure S2: Normal mode vectors of neutral 9-deazaguanine. Structure of 9-deazaguanine was computed using dispersion corrected density functional theory in Gaussian 09. WB97XD/6-31G $(d, p)$ level of theory was used for the purpose. Wavenumber calculations were performed on the optimized structure using same level of theory.*represents the wavenumber obtained in UVRR experiment. 

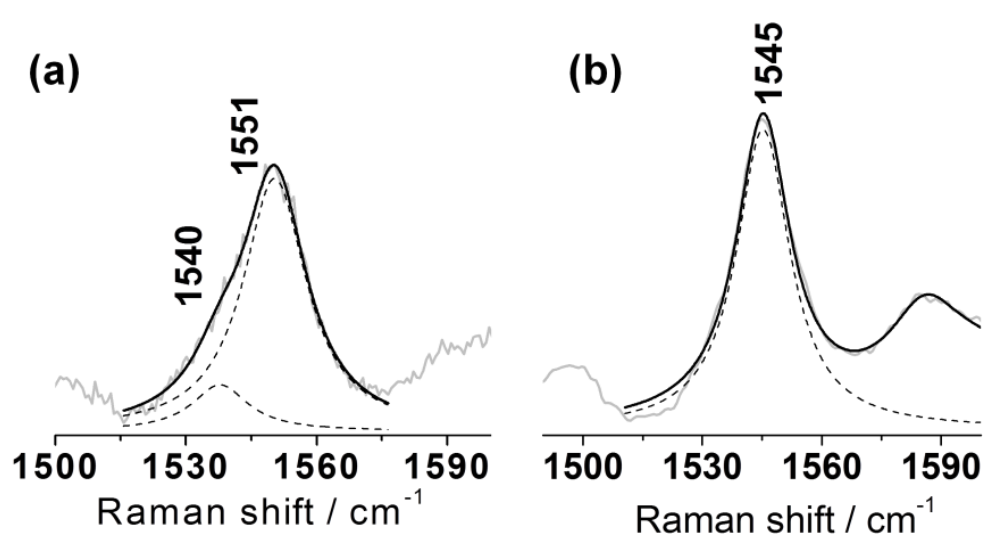

Figure S3: Lorentzian fits to the Raman bands at $1540 \mathrm{~cm}^{-1}$ and $1551 \mathrm{~cm}^{-1}$. (a) The spectrum in the range $1530-1560 \mathrm{~cm}^{-1}$ is fitted with two Lorentzian bands. The experimental spectrum is shown in grey, the fitted spectrum is shown in solid black and the deconvoluted fits to contributing bands are shown in dashed lines. (b) Upon isotopic labeling of hydrogen with deuterium, the band at $1551 \mathrm{~cm}^{-1}$ downshifts to $1545 \mathrm{~cm}^{-1}$ while the band at $1540 \mathrm{~cm}^{-1}$ shifts much lower to $1156 \mathrm{~cm}^{-1}$, out of this range. 


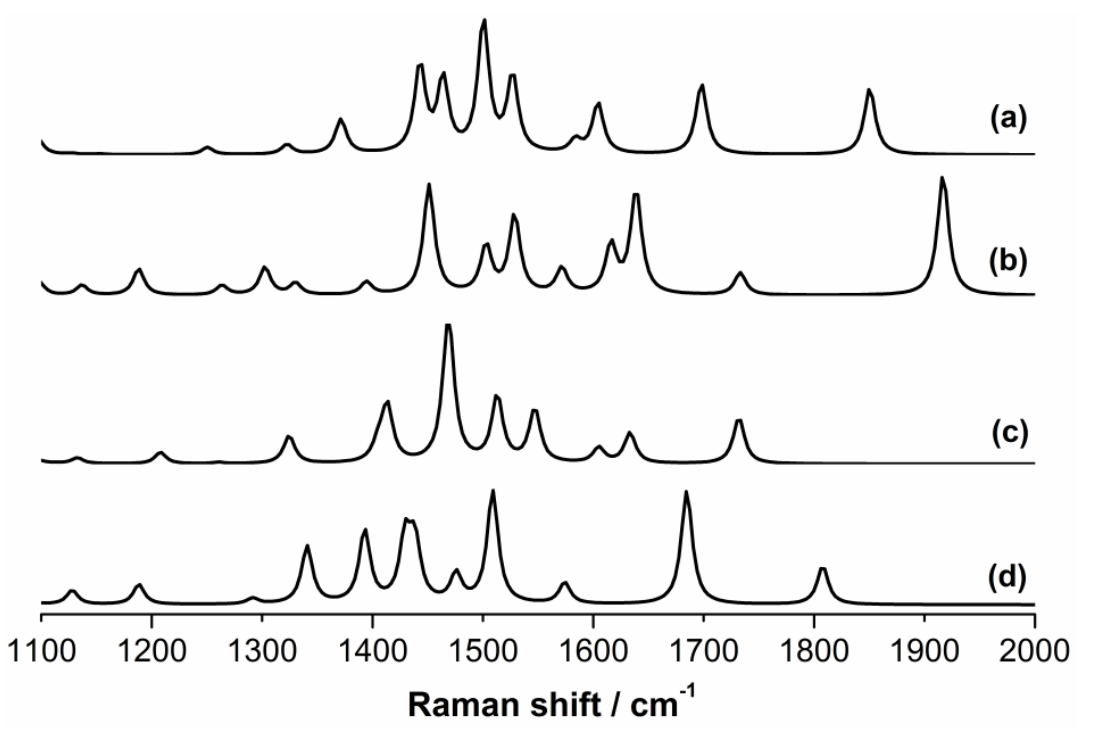

Figure S4: DFT (B3LYP/6-31G (d, p) computed spectra of (a) neutral 9DAH, (b) N3 protonated, (c) N1 deprotonated, (d) N7 deprotonated. N7 deprotonated shows weak carbonyl stretch at 1810 $\mathrm{cm}^{-1}$ whereas in $\mathrm{N} 1$ deprotonated, the carbonyl stretch is completely disappeared. 


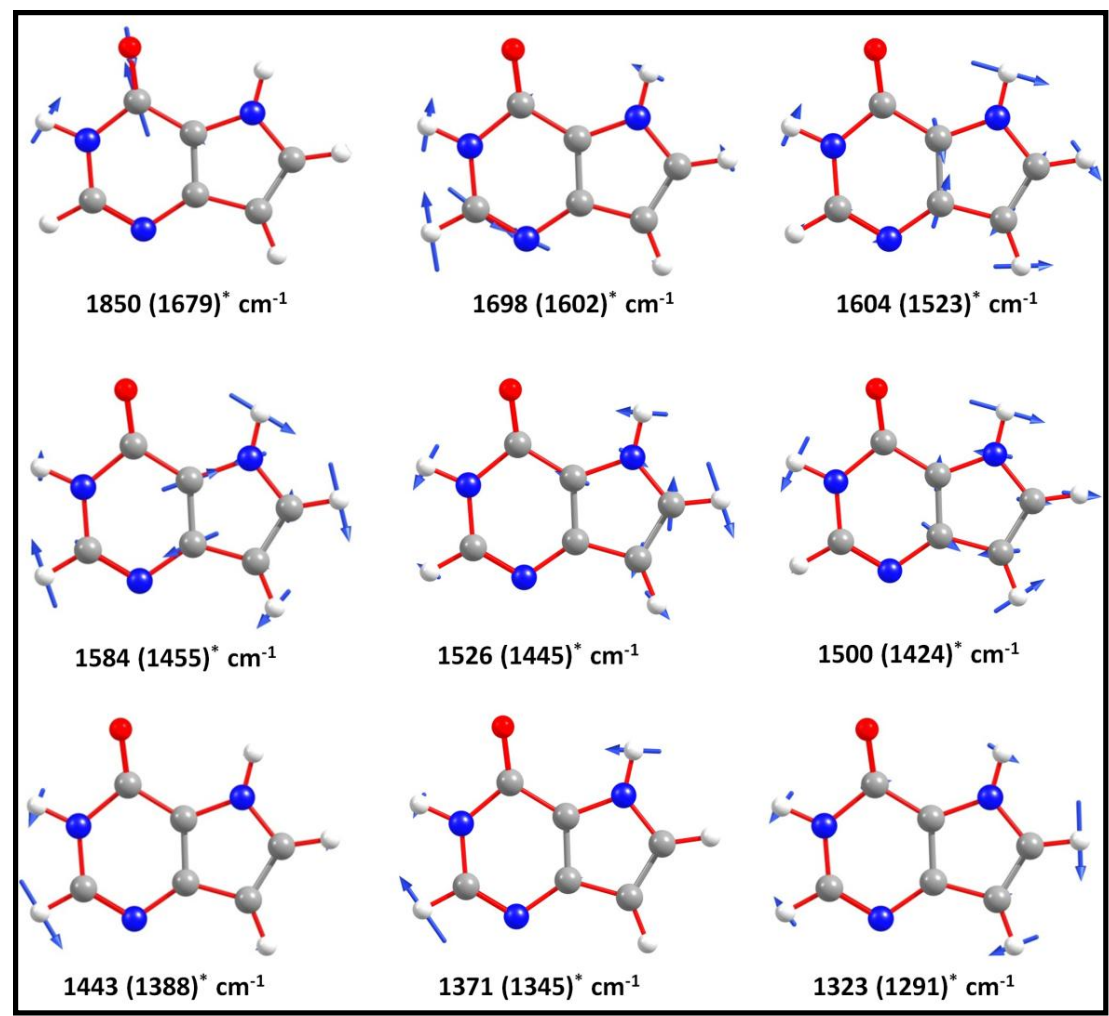

Figure S5: Normal mode vectors of 9-deazahypoxanthine. Structure of 9-deazahypoxanthine was computed using dispersion corrected density functional theory in Gaussian 09. WB97XD/6-31G (d, p) level of theory was used for the purpose. Wavenumber calculations were performed on the optimized structure using same level of theory.*represents the wavenumber obtained in UVRR experiment. 


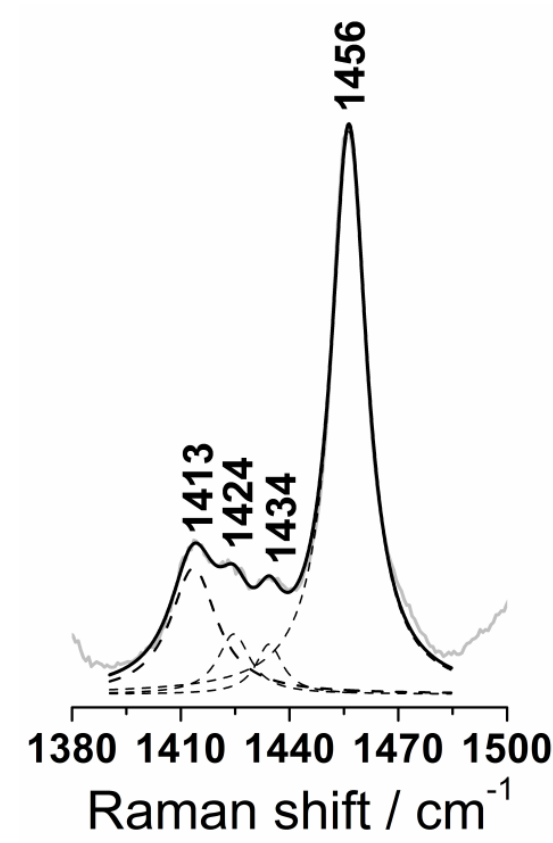

Figure S6: Lorentzian fits to the Raman bands in $1400 \mathrm{~cm}^{-1}$ to $1500 \mathrm{~cm}^{-1}$ region. The experimental spectrum is shown in grey, the fitted spectrum is shown in solid black and the deconvoluted bands are shown in dashed lines. 